\title{
Observations of the Diurnal Dependence of the High-Latitude $F$ Region Ion Density by DMSP Satellites
}

\author{
J. J. SOJKA, W. J. RAITT, AND R. W. SCHUNK
}

Center for Atmospheric and Space Sciences, Utah State University, Logan, Utah 84322

\author{
F. J. RICH AND R. C. SAGALYN
}

Air Force Geophysics Laboratory, Hanscom Air Force Base, Bedford, Massachusetts 01731

\begin{abstract}
Data from the DMSP F2 and F4 satellites for the period December 5-10, 1979, have been used to study the diurnal dependence of the high-latitude ion density at $800-\mathrm{km}$ altitude. A 24-hour periodicity in the minimum orbital density (MOD) during a crossing of the high-latitude region is observed in both the winter and summer hemispheres. The phase of the variation in MOD is such that it has a minimum during the 24-hour period between 0700 and 0900 UT. Both the long term variation of the high-latitude ion density on a time scale of days, and the orbit by orbit variations at the same geomagnetic location in the northern (winter) hemisphere for the magnetically quiet time period chosen show good qualitative agreement with the diurnal dependence predicted by a theoretical model of the ionospheric density at high latitudes under conditions of low convection speeds (Sojka et al., 1981a).
\end{abstract}

\section{INTRODUCTION}

The diurnal variations of plasma density in the polar regions are complex and have been found to exhibit a significant degree of universal time (UT) control [Duncan, 1962; King et al., 1968; Stromman and Maehlum, 1974]. These early measurements were made by ground-based ionosondes situated in the polar regions; each station measured the diurnal variation of a particular ionospheric parameter, such as $f_{o} F_{2}$ [see Duncan, 1962].

Duncan [1962] using data from six circumpolar stations situated in the Antarctic, showed that the diurnal variation of $f_{o} F_{2}$ does not always maximize at local noon, but instead generally peaks between 0300 and 0600 UT. Duncan noted that in this UT range the southern magnetic pole reaches its most sunward location, and he conjectured that if the soft particle precipitation maximized in this time range for this special geometry it could explain the observed increase in the $F$ region ionization. An alternative theory, based on a global-neutral wind system, was advanced by King et al. [1968]. They suggested that a neutral wind induced change in the vertical ionization drift could increase the lifetime of the ions and thereby enhance the electron density in the $F$ region. Challinor [1970] examined the diurnal variations of $f_{o} F_{2}$ at eight Arctic stations and concluded that the neutral wind theory could account for some of the observed temporal and spatial variations. More recently, however, Stromman and Maehlum [1974] showed that neither the Duncan [1962] nor the King et al. [1968] theory can account for the periodic UT variations observed in the Arctic polar region. Similar problems have been experienced in interpreting $f_{o} F_{2}$ data obtained from satellite topside sounder experiments [Muldrew, 1965]. Even with complementary satellite data sets, the full diurnal and LT dependence of the high-latitude ionosphere has not been resolved.

At this point it is useful to distinguish between LT

Copyright $\odot 1982$ by the American Geophysical Union.

Paper number $1 \mathrm{~A} 1670$.

0148-0227/82/001A-1670\$01.00 and diurnal variations. For a given set of ground stations at the same geographic latitude but at different geographic longitudes, a purely LT variation of say $f_{o} F_{2}$ would require that each station observe the same 24 -hour variation. However, if the different stations observe different 24-hour variations of $f_{o} F_{2}$, then the longitude of the station is important and a diurnal variation is present. This diurnal variation corresponds to what Duncan [1962] called the UT control of the polar ionosphere. It should be noted that a diurnal variation can also occur in a magnetic reference frame. In this case, a diurnal variation is evident if the magnetic local time (MLT) variation of a given ionospheric parameter is different at different magnetic longitudes but at the same magnetic latitude.

Recently, we have developed a comprehensive model of the high-latitude ionosphere that displays both LT and diurnal variations in both the geomagnetic and geographic reference frames. This model was obtained by combining a plasma convection model with an ionospheric-atmospheric composition model. The plasma convection model, which is based on the work of Volland [1978], includes an offset between the geographic and geomagnetic poles, the projection of the convection electric field to a circle centered $5^{\circ}$ in the antisunward direction from the magnetic pole, and the tendency of the plasma to corotate about the geographic pole. Equatorward of the circular projection, the electric potential diminishes radially, varying inversely as the fourth power of the magnetic colatitude. The ionospheric-atmospheric composition model includes a wide range of high-latitude processes; complete details are given by Schunk and Raitt [1980] and Sojka et al. [1981a].

The diurnal variation in our model results from the displacement between the geomagnetic and geographic poles. In the geographic frame the entire convection pattern rotates about the geographic pole while continually pointing toward the sun. Therefore the plasma convection velocities should display a diurnal variation when observed in the geographic frame. This model prediction has been verified in a recent comparison between model 

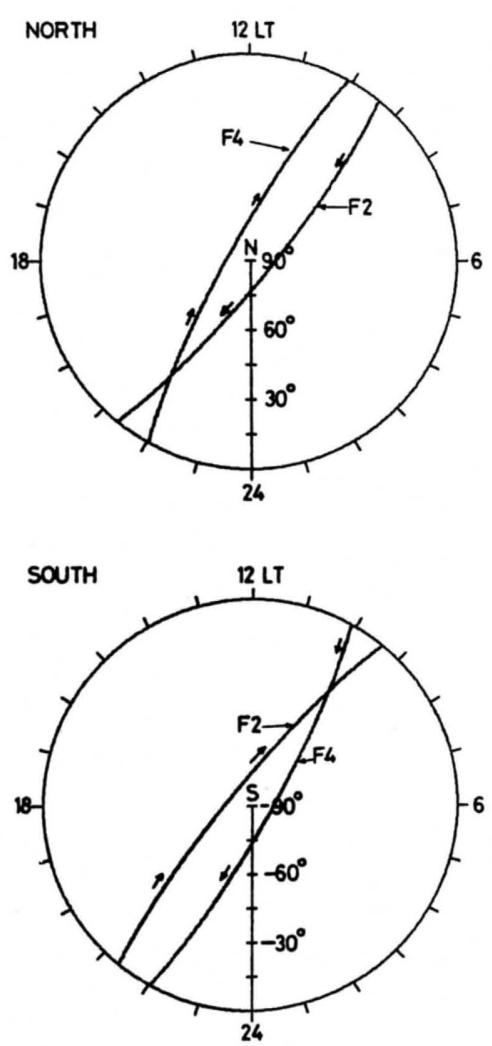

Fig. 1. DMSP sub-satellite tracks for F2 and F4 in geographic latitude-local time coordinates for the period December 5-9, 1979. The top panel shows the sub-satellite tracks in the northern hemisphere, while the bottom panel shows the southern hemisphere. Local time is marked at one hour intervals around the equator and latitude is shown by tick marks at $15^{\circ}$ intervals on the vertical axis. The arrows indicate the direction of the two satellites.

velocities and those measured by the Chatanika and Millstone Hill incoherent scatter radars [Sojka et al., 1980].

The electron density and ion composition also display a marked diurnal variation in both the geographic and geomagnetic reference frames owing to the rotation of the convection pattern about the geographic pole. As this pattern rotates, the high-latitude ionosphere moves toward and then away from the sun, producing a diurnal variation in the photoionization rate. We have successfully compared the major features in electron density and ion composition that are obtained from our model with densities measured by the AE satellites [Sojka et al., $1981 a, b$ ]. Unfortunately, the AE data were not organized with respect to UT, and therefore the large diurnal variation of electron density that our model predicts could not be tested.

In this paper we use the sun synchronous (fixed LT) nature and high time resolution of the ion density measurements from the DMSP F2 and F4 satellites to study the diurnal dependence of in situ ion densities and to compare the diurnal dependence with that predicted by our model. The in situ ion density measurements show a clear diurnal variation repeated over a period of several days. This repetition enables us to separate nondiurnal variations associated with substorm activity from the diurnal variation. In the geographic frame, the sun syn- chronous satellite orbit always crosses a given latitude at the same local times; hence any 24-hour variation found in this frame is a diurnal variation. A more careful selection of orbits is necessary to show the presence of a diurnal variation in the magnetic frame, because at a fixed magnetic latitude MLT varies during a 24-hour period. However, as we show below, the in situ ion density measurements display a clear diurnal variation in both the geographic and geomagnetic reference frames. The observed diurnal variation is in good qualitative agreement with the predictions of the theoretical model.

\section{DMSP F2 AND F4}

In this study we used thermal ion density data from the defense meteorological satellite program (DMSP) satellites 2 and 4 (F2 and F4) of the Block 5D series. These data were collected by a planar retarding potential analyzer, a part of the topside ionosphere plasma monitor flown on these two satellites. A full description of the instrument is given by Smiddy et al. [1978], while the inflight operation of the instrument has been reported by Rich et al. [1980]. Both satellite orbits are nearly sun synchronous with a period of $101.5 \mathrm{~min}$, inclinations of $99.1^{\circ}$ and $98.7^{\circ}$, and altitudes of $820 \mathrm{~km}$ and $830 \mathrm{~km}$ for F2 and F4, respectively. The ascending nodes of the two orbits were separated by approximately 12 hours in local time at the epoch being studied. In Figure 1, the sub-satellite tracks for F2 and F4 are presented for the period December 5-10, 1979. The two panels of Figure 1 show the sub-satellite tracks plotted on a polar diagram. The centers of the diagrams represent the geographic pole, north (N) for the upper panel and south (S) for the lower panel. Our southern hemisphere projection (lower panel) is drawn as if the sub-satellite track was viewed through the earth, that is, both northern and southern tracks are viewed from the same point in space above the north pole. The F2 orbit has its ascending node at 0930 hour LT, while the F4 orbit has its ascending node at 2200 hour LT. Thus, both satellites lie approximately in the 1000-2200 hour local time plane. However, because of the nearly 12 hours difference of local time in the ascending nodes and the nearly equal inclinations, the sub-satellite paths of the two satellites are separated by $17.4^{\circ}$ arc over the polar regions and can be regarded as being fixed in the geographic local time frame.

In the magnetic local time frame the orbits are not fixed. As a consequence of the offset between the geomagnetic and geographic poles, the sub-satellite tracks in the magnetic local time frame vary with universal time (UT) as the earth rotates. The offset between the poles is approximately $11.5^{\circ}$ in the northern hemisphere and $15^{\circ}$ in the southern hemisphere. In Figure 2, sections of the sub-satellite tracks in the northern hemisphere are shown in the magnetic local time frame over a period of 24 hours. Each panel shows a total of 14 tracks plotted as a sequence of dots in the polar diagram. The center of the polar diagram corresponds to the magnetic north pole. In the left panel, the tracks are associated with the F4 satellite, while the right panel shows F2 tracks. Clearly, the two satellites cover a large portion of the magnetic local time-high latitude region in a 24-hour period. In 

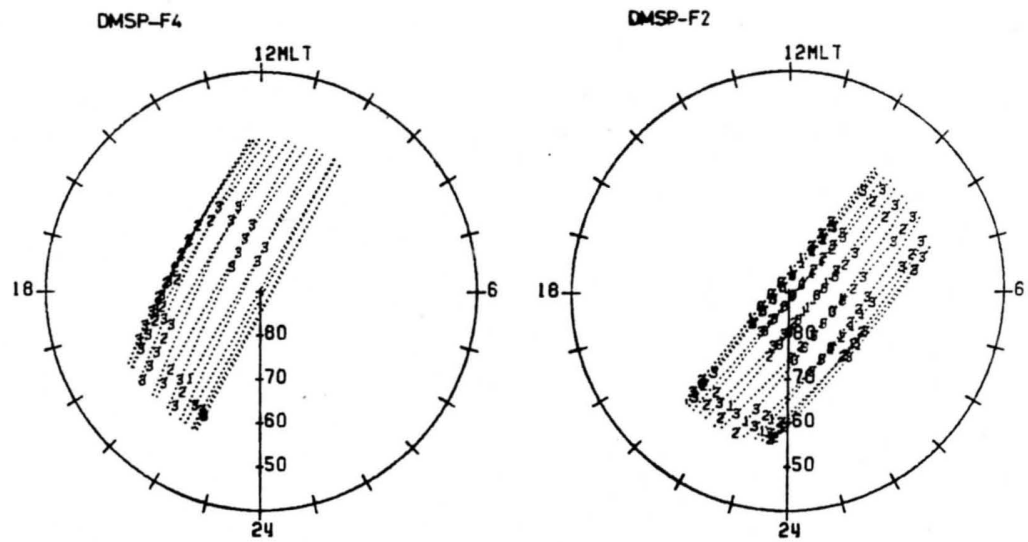

Fig. 2. DMSP sub-satellilte tracks for F4 (left panel) and F2 (right panel) in geomagnetic latitude-local time coordinates in the northern hemisphere for a 24-hour period in December, 1979. Magnetic local time is marked at one hour intervals around the $40^{\circ}$ magnetic latitude circle. Magnetic latitude is shown by tick marks at $10^{\circ}$ intervals on the vertical axis. The numbers along the tracks are used to display the regions of low plasma density, as predicted by the theoretical model of Sojka et al., [1981a]. The number 3 corresponds to densities less than $2 \times 10^{2} \mathrm{~cm}^{-3}$, while the number 0 corresponds to densities less than $20 \mathrm{~cm}^{-3}$.

the southern hemisphere, where the dipole offset is larger, the area covered by the orbits is even larger [see Gussenhoven et al., 1980]. Northern hemisphere MLT coverage between the two satellites overlaps in the 0930 to 2130 MLT plane; however, the two satellites do not cross this region at the same UT. This region is crossed by F2 at about 0900 UT and by F4 at 2100 UT.

\section{The High-LATItude DiURNAL EFFECT IN THE MAGNETIC FRAME}

We now consider individual orbits over the winter (northern) hemisphere where marked diurnal variations are predicted over a large part of the high latitude region in the magnetic frame. Figure 2 shows how the two satellites, F2 and F4, cover an overlapping region in the magnetic local time frame. The satellites cross this common region at 12-hour intervals. From our model calculations, these two satellites are expected to see different densities over the polar cap, with F4 observing higher densities by almost an order of magnitude. Figure 3 shows two winter (northern) hemisphere passes along the 0930-2130 MLT meridian, the region common to F2 and F4. The crosses indicate data from F2, while the squares indicate data from F4. Density is plotted against magnetic latitude from the sunlit equator (ascending node from DMSP F2) to the magnetic pole $\left(90^{\circ}\right)$ and then back to the equator in darkness (descending node for DMSP F2). In general, the F4 densities are larger than those for F2 in the polar regions. At $75^{\circ}$ magnetic latitude in the descending node section of Figure 3, the density difference between the two satellites reaches an order of magnitude. This difference is present during the four day data period for all orbits that lie along the overlapping region. The effect of structured auroral precipitation is not very marked, since the data period selected was for low $K p$ (see lower panel in Figure 7). There are, however, fine scale variations in density on time scales of tens of seconds in which the density varies by a factor of about 2 . These high latitude variations are associated with the dynamic low intensity auroral fluctuations.
In Figure 4, density data from two DMSP F2 orbits, which cover the same region of the magnetic local time frame, are plotted. These two orbits correspond to the two orbits which pass nearest the $80^{\circ}$ latitude tick mark in Figure 2 (right panel) and are separated by 8 hours in UT. For these orbits a marked diurnal effect is predicted and is observed (see Figure 4). In the ascending node section of Figure 4, the orbit shown by square symbols has the larger densities; however, on crossing the pole and going toward the equator, the density for this orbit decreases to such an extent that it is significantly lower than the density observed along the second orbit. Superimposed upon this general density variation, which is the predicted diurnal effect, there are finer density variations, again attributable to dynamic low $K p$ auroral activity.

\section{DMSP-F2/4 NORTHERN HEMISFHERE (WINTER)}

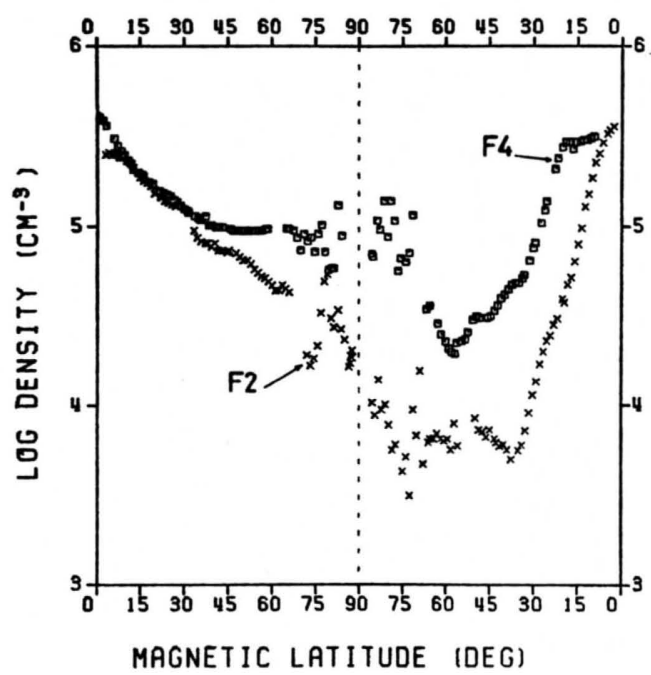

Fig. 3. A comparison between the ion densities measured along the F2 and F4 satellite tracks in the northern hemisphere as a function of magnetic latitude. The two orbits selected lie in approximately the same location in the geomagnetic local time frame (see Figure 2), but the traversals are separated by approximately 12 hours. 


\section{DMSP-F2 NORTHERN HEMISPHERE (WINTER)}

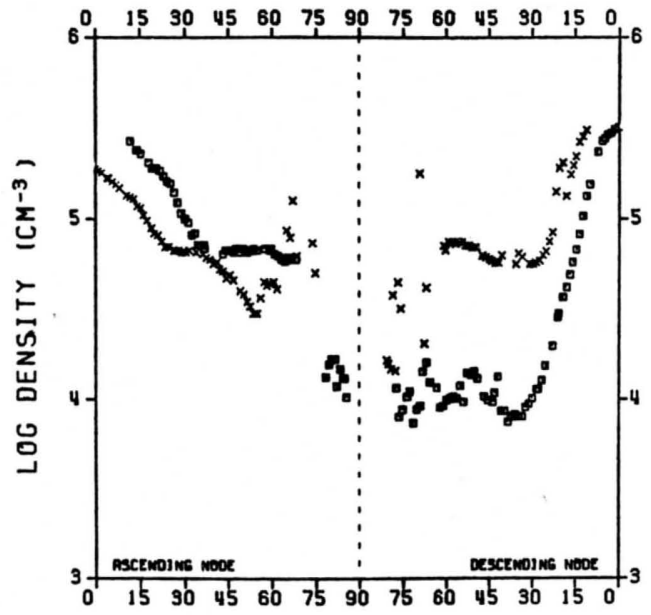

MAGNETIC LATITUDE (DEG)

Fig. 4. A comparison between the ion densities measured along two traversals of the northern hemisphere by the F2 satellite as a function of magnetic latitude. The two orbits selected lie in approximately the same location in the geomagnetic local time frame, but the traversals are separated by approximately 8 hours.

\section{Model Ion Densities}

The time difference between the times that the satellites cross the same region in the magnetic frame is particularly useful, since the data obtained can be used to test our recent model prediction that the high-latitude ionosphere displays a significant diurnal variation in the magnetic frame as well as geographic frame owing to the displacement between the geomagnetic and geographic poles [Sojka et al., 1979, 1981a, b]. Our model predicts that at a given region in the high-latitude ionosphere, specified by a latitude and local time in either reference frame, the electron density can vary with UT by as much as an order of magnitude. Examples of the distribution of electron density over the polar region at $300 \mathrm{~km}$ for four different UT's for geomagnetically guiet conditions are presented by Sojka et al. [1981b]. The features displayed at $800 \mathrm{~km}$ are similar to those at $300 \mathrm{~km}$, but the absolute electron density values are lower at the higher altitude.

Our previous model study was for the northern hemisphere at solar minimum, winter, and low geomagnetic activity [Sojka et al., 1981a]. The DMSP data available were taken in both the northern and southern hemispheres from a time period corresponding to low geomagnetic activity in December 1979. However, the data were collected during an extremely active solar maximum phase [cf. Torr et al., 1979], while the model corresponds to solar minimum conditions. Because of this difference in solar activity, a comparison of absolute densities between the model and the data is not possible, owing to the expected difference in neutral atmospheres and EUV ionization production rates [cf. Schunk and Raitt, 1980]. Nevertheless, the relative diurnal modulation and phase should be similar for the model and the data, since the diurnal effect depends on the relative motions of the terminator, the neutral wind, the auroral oval, and the field tubes of plasma; these relative motions are similar for solar maximum and solar minimum.

To facilitate the comparison between the model and data, the model $\mathrm{O}^{+}$density will be displayed along the $\mathrm{F}_{2}$ and F4 orbits for a period of 24 hours. In Figure 2, the model densities are indicated along the F2 and F4 subsatellite tracks by the numbers $0,1,2$, and 3 . These numbers are used to show the regions where the $\mathrm{O}^{+}$density at $800 \mathrm{~km}$ falls below $200 \mathrm{~cm}^{-3}$. Number 3 cor. responds to densities between 80 and $200 \mathrm{~cm}^{-3}$; number 2 corresponds to 40 and $80 \mathrm{~cm}^{-3}$; number 1 corresponds to 20 to $40 \mathrm{~cm}^{-3}$; and number 0 corresponds to densities below $20 \mathrm{~cm}^{-3}$. Density numbers are only shown every third point in Figure 2; where the density is above 200 $\mathrm{cm}^{-3}$ only the dot representing the satellite position is shown. Comparing the densities in Figure 2 left and right panels), where the satellites overlap in the MLT frame, shows an order of magnitude change in $\mathrm{O}^{+}$density with UT. Our model predicts that DMSP F2 should measure $\mathrm{O}^{+}$densities about an order of magnitude lower than DMSP F4 in the polar cap which is consistent and in the same diurnal phase as the DMSP data presented in Figure 3.

The diurnal variation of the model $\mathrm{O}^{+}$density is also observed by a single satellite, since the motion of the subsatellite tracks in the magnetic frame results in overlap during the course of a day. In the right panel of Figure 2, two sub-satellite tracks intersect the vertical axis at approximately $80^{\circ}$ magnetic latitude. At this point, one of the tracks shows a density number ' 3 ', while the second track shows a density number ' 0 '; a factor of 4 difference in $\mathrm{O}^{+}$density at two different UT's but at the same magnetic location. This diurnal dependence is again the same as that observed and shown in Figure 4.

\section{DMSP THERMAL ION DENSITIES IN THE GEOGRAPHIC FRAME}

Data for the period December 5-9, 1979, were acquired from the thermal ion experiment on DMSP F2 and F4. A thermal ion density measurement was made approximately each $1 / 7 \mathrm{~s}$ during this period. To get an overview of the density variation, the density samples were averaged over 17-s intervals (approximately $1^{\circ}$ in latitude). Since the southern polar region satellite passes were in sunlight (summer) and the northern polar region passes were in darkness (winter), large density differences were found. In Figure 5, the ion density for the two satellites and two hemispheres have been plotted sequentially in UT. The upper two panels show the winter hemisphere data, with the DMSP F2 data in the top panel. Summer data are shown in the lower two panels, again with DMSP F2 being the upper of these two panels. Density is plotted on a logarithmic scale over the range $10^{3}$ to $10^{6} \mathrm{~cm}^{-3}$. Each panel shows a marked variation of density as the satellite crosses the hemisphere; the winter hemisphere shows over 2 orders of magnitude density change per orbit, while the summer hemisphere shows about 1 order of magnitude density change. In the winter hemisphere, the lowest density is almost an order of magnitude lower than that in the summer hemisphere; the low density regions are associated with deep troughs and polar cap density depletions. 

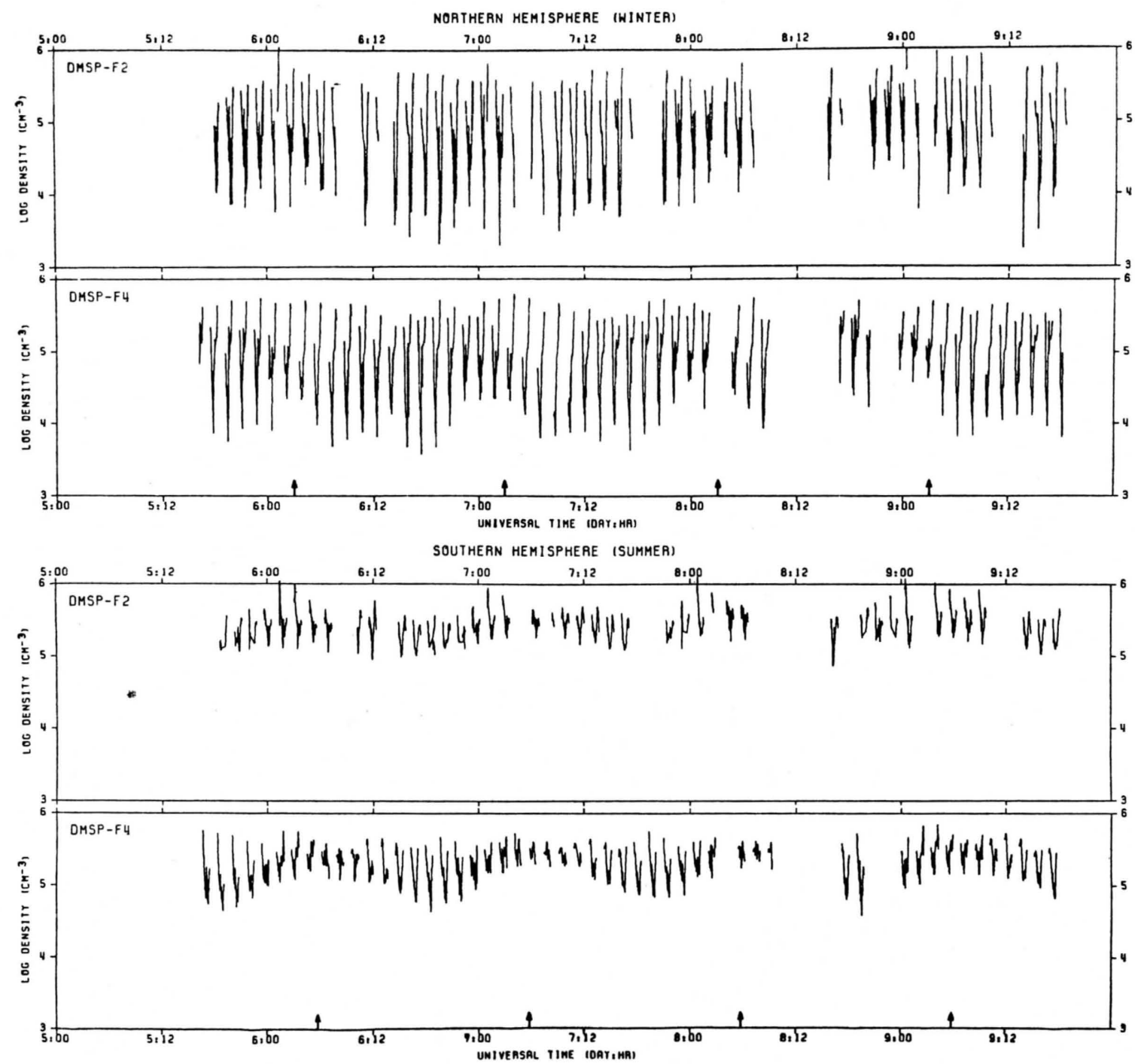

Fig. 5. Plots of ionospheric ion density at $800 \mathrm{~km}$ versus UT for the four days, December 5-9, 1979. The upper panels show northern hemisphere data from DMSP-F2 and DMSP-F4, respectively, while the lower panels show southern hemisphere data. For the DMSP-F4 data, arrows are shown at 24-hour intervals to highlight the diurnal effect, as discussed in the text.

Figures 3 and 4 show four northern hemisphere orbits; in each case the lowest densities are found in the dark sector with well-defined mid-latitude trough minima. On each DMSP 2 and 4 orbit such a dark sector lowest density is present. Our model predicts that this lowest density, although occurring at the same LT for the sun synchronous orbits, but not at the same geomagnetic local time, should show a diurnal variation. In the subsequent sections, we shall define the lowest dark sector density for each orbit as the minimum orbital density (MOD) and study its diurnal behavior.

This minimum orbital density (MOD) varies significantly from orbit to orbit. The winter hemisphere variation of the MOD value appears somewhat random, while in the summer hemisphere a more systematic variation is apparent. From the bottom panel in Figure 5, it can be seen that the F4 MOD values show a periodic variation. An arrow has been placed at the UT of the first maximum MOD and then three other arrows at 24-hour intervals. These subsequent arrows align with subsequent maxima in the MOD modulation, indicating that it is a 24-hour diurnal variation in the geographic frame, since in this frame the LT of the satellite is fixed. In the second lowest panel of Figure 5, summer F2 data, the diurnal modulation is again present, but its characteristics are different from those observed by F4 owing to the different geographic locations of the two satellites. In the winter hemisphere the F4 satellite data also show a diurnal variation; however, in this case it is rather complex. Arrows have been placed at 24-hour UT intervals from the first maximum in MOD in the F4 panel (second panel from the top in Figure 5). A comparison of the variation between arrows in the MOD again reveals a repetitive pattern with a 24 -hour period.

\section{THE WiNTER High LATITUDE REgion}

Figure 6 (four lowest panels) shows the daily F4 variation of the MOD plotted on a logarithmic scale for the 24-hour periods 6, 7, 8, and 9 of December 1979. These MOD values correspond to the F4 winter hemisphere data shown in the second from the top panel of Figure 5. 
DMSP-FY NORTHERN HEMISPHERE.

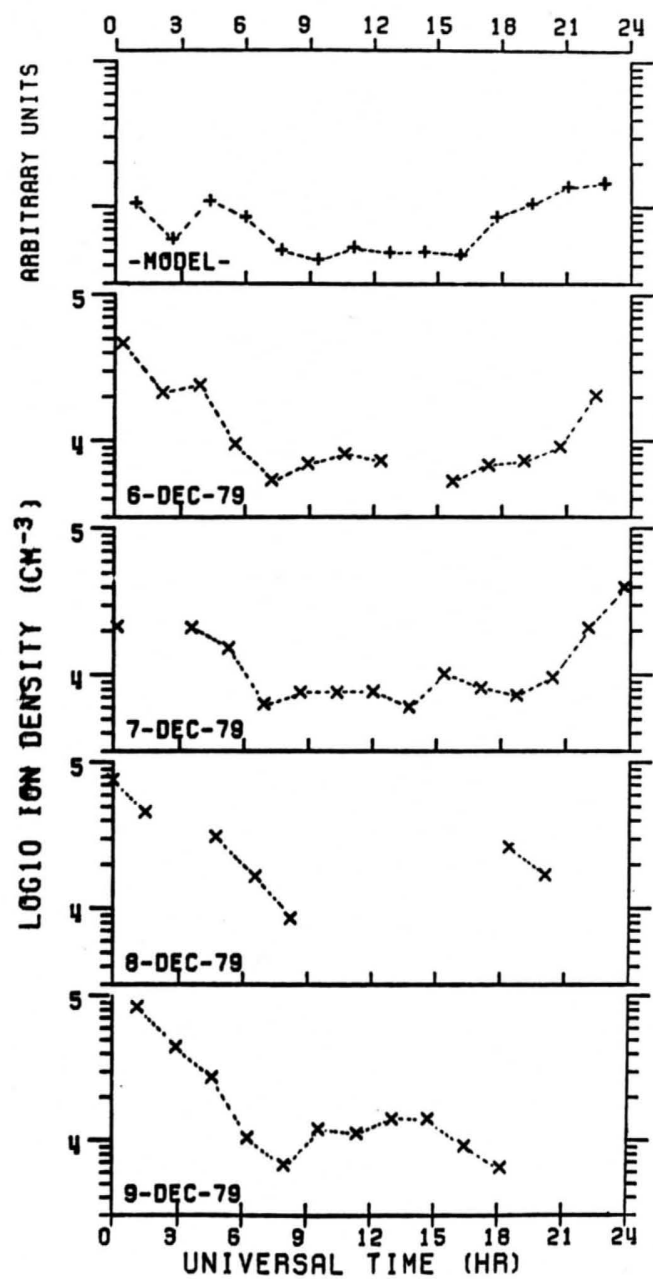

Fig. 6. The minimum ion density for each traversal of the northern hemisphere by DMSP-F4 as a function of universal time. The lower four panels correspond to measurements made during the four days indicated. The top panel shows the corresponding diurnal dependence as predicted by the Sojka et al. [1981a] model; the density units are arbitrary.

The top panel of Figure 6 shows the predicted F4 MOD values, again plotted logarithmically, from the model study of Sojka et al., [1981a]. In each panel of Figure 6, the symbol, either an $x$ or + , represents the minimum density encountered in a northern hemisphere pass by the satellite DMSP F4. The symbols, which are joined by a dashed line, indicate that these MOD values are from consecutive orbits. A large data gap exists on the 8th of December where data was missing in the MOD latitude regions, although not at lower latitudes. Comparing the four data sets reveals a common variation; as UT increases, the MOD decreases reaching a minimum between 0700 and 0900 UT. From this UT, the MOD value increases slightly until it encounters a second minimum between 1400 and 1800 UT. After this UT, the MOD continually increases. This behavior is generally similar to the model variation displayed in the top panel of Figure 6, which shows a broad minimum between 0900 and 1000 UT followed by a slight increase and a second minimum at $1600 \mathrm{UT}$, from which time on the MOD value increases. Thus the general form of the computed and measured diurnal variations compare favorably. However, the absolute values cannot be compared because of the different phases of the solar cycle used for the model calculation and existing during the observation period.

The variation in the F2 MOD shown in Figure 5 does not have such a clear diurnal pattern as the F4 MOD variation. However, when we plot the F2 MOD for the winter hemisphere over the five day period together with the $K p$ index (Figure 7), we find a tendency for the F2 MOD to positively correlate with the geomagnetic activity index. In Figure 7, the $K p$ and MOD values remain relatively constant until 1200 UT on December 6, at which time both decrease and remain low until 1200 UT on December 7. From this time, both the MOD and $K p$ increase, reaching a maximum at about 1500 UT on December 8.

The dominance of geomagnetic activity effects over diurnal effects for the F2 satellite winter data set has not been predicted by the model because the model has only been run for two cases; an idealized quiet time and a storm time. The real world, as described by the data, undergoes a continuous range of configurations as the geomagnetic activity changes. In particular, the following factors need to be considered when comparing the model and the data:

1. The convection electric field strength across the high latitude ionosphere is positively correlated to $K p$ [Heppner, 1973].

2. The size of the auroral oval increases with $K p$ [Gussenhoven et al., 1981].

3. The number flux and average energy of precipitating particles tends to increase with geomagnetic activity [Spiro et al., 1980].

4. The diffuse precipitation of particles in the polar cap, or polar rain, is a source of ionization not included in the model; however, the intensity of the polar rain does not correlate well with $K p$ (D. Hardy, private communication, 1981).

The model can give us important clues as to why a $K p$ effect should be more pronounced in the F2 data than the F4 data. The lower average MOD predicted for the F2 data (Figure 2) means that small changes in the absolute ionization levels will have a larger relative effect in the F2 than the F4 data. We also note that the MOD for the F2 orbit in the model and in the data occurs almost always in the polar cap, while the MOD for the F4 orbit occurs in the midlatitude trough region. The minimum density in the trough is largely determined by a balance between the corotation electric field and the convection electric field causing the stagnation point. As the convection electric field increases in strength, the stagnation point moves equatorward, but the time for a particle to drift passed the stagnation point is relatively unchanged. The minimum density in the polar cap is largely determined by the speed with which particles can cross the polar cap from the dayside auroral zone to the nightside auroral zone. Thus, it is reasonable to expect a greater dependence on geomagnetic activity of the MOD in the F2 data than in the F4 data. 

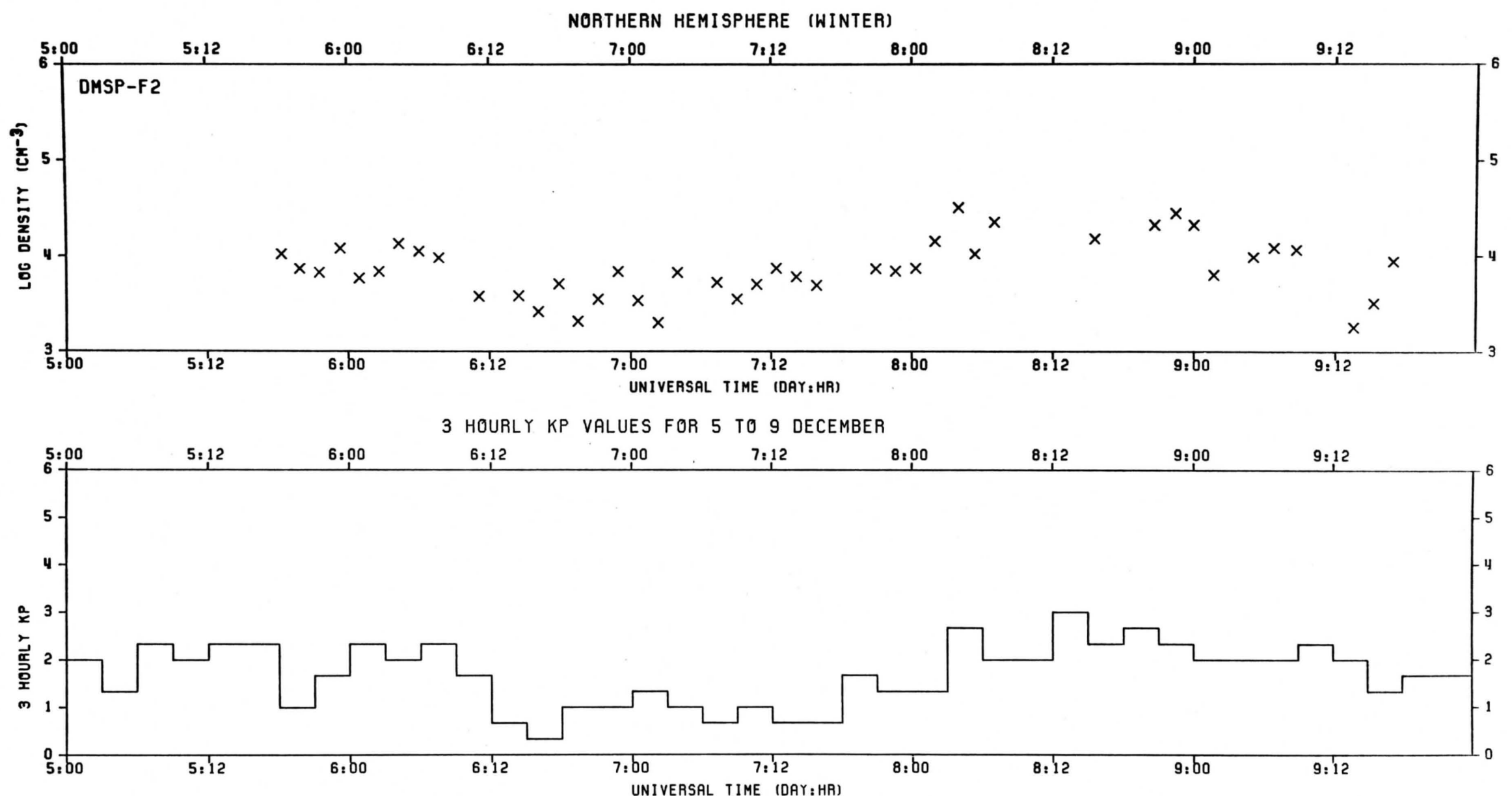

Fig. 7. A comparison between the minimum ion density measured on each traversal of the northern hemisphere by DMSP-F2 (upper panel) and the variation of the 3-hour $K p$ index (lower panel), both as a function of universal time. 


\section{SUMMARY}

We studied the diurnal variation of measured ion density at approximately $800 \mathrm{~km}$ in both the geographic and geomagnetic frames for the time period December 5-9, 1979 , and compared it with predictions from a highlatitude ionosopheric model [Sojka et al., 1981a]. The data set was collected from the DMSP satellites designated F2 and F4, which were in near polar sun synchronous orbits, and therefore collected data from both summer and winter high-latitude zones for the time period analyzed. In addition, the two satellites were in a similar time plane (1000-2200 hours), but their ascending nodes were separated by 12 hours in local time.

In order to study the diurnal effect in the geomagnetic frame, carefully selected individual orbits were used. The effect was demonstrated by comparing the ion density along the satellite track for cases where the orbits covered very nearly the same position in the magnetic latitude-magnetic local time frame. It was possible to use both orbits from the two satellites and separate orbits from the same satellite for the comparison. In each case the data taken were separated by 12 hours in UT. The density variations were found to show marked differences for the pairs of orbits selected, again indicating the diurnal effect. The variations seen were in good qualitative agreement with our model; however, as explained in the text, quantitative comparisons were not possible due to the widely different solar activities between those used for the model calculations and the value pertaining to the measurement period.

In the geographic frame we identified a characteristic of the high latitude traverses that we called the minimum orbital density (MOD), which showed a diurnal dependence with a clear 24-hour period both in the summer hemisphere and in the winter hemisphere. Owing to the 12-hour local time shift between ascending nodes, the two satellites cover different regions in magnetic latitude-local time space. Both the model predictions and the measurements indicate that this results in generally lower, high latitude, winter ion densities for F2 in comparison with F4; thus, the diurnal effect is less pronounced for F2 than F4 due to the effect of variable, diffuse, low-intensity particle precipitation in monitoring the MOD.

The variation of the low intensity precipitation mentioned above is thought to be the reason for a marked dependence of MOD on $K p$, which can be seen most clearly in the F2 ion density data. The correlation between $K p$ and MOD is positive for the winter hemisphere for the time period studied.

Both on the time scale of several days and on the individual orbit scale, we see a marked diurnal dependence of ion densities at $800 \mathrm{~km}$ in both the geographic and geomagnetic reference frames for the time period December 5-9, 1979. These variations are consistent with the predictions of our high latitude ionospheric model [Sojka et al., 1981a].

Acknowledgments. This research was supported by Air Force contract USAF/ESD F19628-79-C-0025, NASA grant
NAGW-77, and NSF grant ATM80-15497 to Utah State University.

\section{REFERENCES}

Challinor, R. A., The behavior of the Arctic $F$ region in winter, J. Atmos. Terr. Phys., 32, 1959, 1970.

Duncan, P. A., Universal-time control of the Arctic and Antarctic F region, J. Geophys. Res., 67., 1823, 1962.

Gussenhoven, M. S., D. A. Hardy, and W. J. Burke, Comment on diurnal variation of the auroral oval size by C.-I. Meng, J. Geophys. Res., 85, 2373-2374, 1980.

Gussenhoven, M. S., D. A. Hardy, and W. J. Burke, DMSP/F2 electron observations of equatorward auroral boundaries and their relationship to magnetospheric electric fields, J. Geophys. Res., 86, 768-778, 1981.

Heppner, J. P., High latitude electric fields and the modulations related to interplanetary magnetic field parameters, Radio Sci., 8, 933, 1973.

King, J. W., H. Kohl, D. M. Preece, and C. Seabrook, An explanation of phenomena occurring in the high-latitude ionosphere at certain Universal Times, J. Atmos. Terr. Phys., 30, 11, 1968.

Muldrew, D. B., $F$ layer ionization trough deduced from Alouette data, J. Geophys. Res., 70, 2635, 1965.

Rich, F., M. Smiddy, R. C. Sagalyn, W. J. Burke, P. Anderson, S. Bredesen, and W. P. Sullivan, In-flight characteristics of the topside ionospheric monitor (SSIE) on the DMSP satellite flight 2 and flight 4, Rep. $A F G L$ TR-80-0152, Air Force Geophys. Lab., Bedford, Mass., 1980.

Schunk, R. W., and W. J. Raitt, Atomic nitrogen and oxygen ions in the daytime high-latitude $F$ region, $J$. Geophys. Res., 85, 1255-1272, 1980.

Smiddy, M., R. C. Sagalyn, W. P. Sullivan, P. G. L. Wildman, P. Anderson, and F. Rich, The topside ionosphere plasma monitor (SSIE) for the block $5 \mathrm{D} /$ flight 2 satellite, Rep. AFGL-TR-78-0071, Air Force Geophys. Lab., Bedford, Mass., 1978.

Sojka, J. J., W. J. Raitt, and R. W. Schunk, Effect of displaced geomagnetic and geographic poles on high. latitude plasma convection and ionospheric depletions, $J$. Geophys. Res., 84, 5943-5951, 1979.

Sojka, J. J., J. C. Foster, W. J. Raitt, R. W. Schunk, and J. R. Doupnik, High-latitude convection: Comparison of a simple model with incoherent scatter observations, J. Geophys. Res., 85, 703-709, 1980.

Sojka, J. J., W. J. Raitt, and R. W. Schunk, A theoretical study of the high-latitude winter $F$ region at solar minimum for low magnetic activity, J. Geophys. Res., 86, 609-621, $1981 a$.

Sojka, J. J., W. J. Raitt, and R. W. Schunk, Theoretical predictions for ion composition in the high-latitude winter $F$ region for solar minimum and low magnetic activity, $J$. Geophys. Res., 86, 2206-2216, $1981 b$.

Spiro, R. W., L. J. Maher, and P. H. Reiff, Dependence of average precipitating electron flux and derived ionospheric conductivity on geomagnetic activity (abstract), Eos Trans. $A G U, 61,46,1092,1980$.

Stromman, J. R., and B. W. Maehlum, Reconsideration of the diurnal variations in the polar $F$ region ionization, $J$. Atmos. Terr. Phys., 36, 1403, 1974.

Torr, M. R., D. G. Torr, R. A. Ong, and H. E. Hinteregger, Ionization frequencies for major thermospheric constituents as a function of solar cycle 21 , Geophys. Res. Lett., 6 , $771-774,1979$.

Volland, H., A model of the magnetospheric electric convection field, J. Geophys. Res., 83, 2695-2699, 1978.

(Received May 29, 1981; revised October 16, 1981; accepted October 26, 1981.) 